ARTICLE

\title{
A CpG-methylation-based assay to predict survival in clear cell renal cell carcinoma
}

Jin-Huan Wei ${ }^{1, \star}$, Ahmed Haddad ${ }^{2, \star}$, Kai-Jie Wu ${ }^{3, \star}$, Hong-Wei Zhao ${ }^{4, \star}$, Payal Kapur ${ }^{5}$, Zhi-Ling Zhang ${ }^{6}$, Liang-Yun Zhao ${ }^{7}$, Zhen-Hua Chen ${ }^{1}$, Yun-Yun Zhou ${ }^{8}$, Jian-Cheng Zhou ${ }^{2}$, Bin Wang ${ }^{2}$, Yan-Hong Yu ${ }^{7}$, Mu-Yan Cai ${ }^{9}$, Dan Xie ${ }^{9}$, Bing Liao ${ }^{10}$, Cai-Xia Li ${ }^{11}$, Pei-Xing Li" ${ }^{11}$, Zong-Ren Wang ${ }^{1}$, Fang-Jian Zhou ${ }^{6}$, Lei Shi ${ }^{4}$, Qing-Zuo Liu ${ }^{4}$, Zhen-Li Gao ${ }^{4}$, Da-Lin He ${ }^{3}$, Wei Chen ${ }^{1}$, Jer-Tsong Hsieh², Quan-Zhen Li ${ }^{12}$, Vitaly Margulis ${ }^{2}$ \& Jun-Hang Luo ${ }^{1}$

Clear cell renal cell carcinomas (ccRCCs) display divergent clinical behaviours. Molecular markers might improve risk stratification of $c \mathrm{RCC}$. Here we use, based on genome-wide CpG methylation profiling, a LASSO model to develop a five-CpG-based assay for $\mathrm{ccRCC}$ prognosis that can be used with formalin-fixed paraffin-embedded specimens. The five-CpG-based classifier was validated in three independent sets from China, United States and the Cancer Genome Atlas data set. The classifier predicts the overall survival of ccRCC patients (hazard ratio $=2.96-4.82 ; P=3.9 \times 10^{-6}-2.2 \times 10^{-9}$ ), independent of standard clinical prognostic factors. The five-CpG-based classifier successfully categorizes patients into high-risk and low-risk groups, with significant differences of clinical outcome in respective clinical stages and individual 'stage, size, grade and necrosis' scores. Moreover, methylation at the five CpGs correlates with expression of five genes: PITX1, FOXE3, TWF2, EHBP1L1 and RIN1. Our five-CpG-based classifier is a practical and reliable prognostic tool for $c \subset R C C$ that can add prognostic value to the staging system.

\footnotetext{
${ }^{1}$ Department of Urology, First Affiliated Hospital, Sun Yat-sen University, No. 58, ZhongShan Second Road, Guangdong 510080, China. ${ }^{2}$ Department of Urology, University of Texas Southwestern Medical Center at Dallas, Dallas, Texas 75390, USA. ${ }^{3}$ Department of Urology, First Affiliated Hospital of Xi'an Jiaotong University, Shaanxi 710061, China. ${ }^{4}$ Department of Urology, Affiliated Yantai Yuhuangding Hospital, Qingdao University Medical College, Shandong 264000, China. ${ }^{5}$ Department of Pathology, University of Texas Southwestern Medical Center at Dallas, Dallas, Texas 75390, USA. ${ }^{6}$ Department of Urology, Cancer Center, Sun Yat-sen University, Guangdong 510060, China. ${ }^{7}$ Department of Urology, Affiliated Hospital of Kunming University of Science and Technology, Yunnan 650032, China. ${ }^{8}$ Quantitive Biomedical Research Center, University of Texas Southwestern Medical Center at Dallas, Dallas, Texas 75390, USA. ${ }^{9}$ Department of Pathology, Cancer Center, Sun Yat-sen University, Guangdong 510060, China. ${ }^{10}$ Department of Pathology, First Affiliated Hospital, Sun Yat-sen University, Guangdong 510080, China. ${ }^{11}$ School of Mathematics and Computational Science, Sun Yat-sen University, Guangdong 510275, China. ${ }^{12}$ Department of Immunology and Microarray Core, University of Texas Southwestern Medical Center at Dallas, Dallas, Texas 75390, USA. * These authors contributed equally to this work. Correspondence and requests for materials should be addressed to J.H.L. (email: luojunh@mail.sysu.edu.cn).
} 
R enal cell carcinoma (RCC) is the most common malignant neoplasm arising from the kidney and it represents $\sim 2-3 \%$ of all human malignancies. The major histological subtype is clear cell RCC (ccRCC), accounting for $80-90 \%$ of all RCC cases $^{1}$. TNM stage and Fuhrman grade remain the most commonly used predictors of clinical outcome for patients with ccRCC. Clinically integrated systems, such as the Mayo Clinic stage, size, grade and necrosis (SSIGN) score and the University of California Integrated Staging System, can improve prognostic accuracy ${ }^{2,3}$. However, patients with similar clinical features or integrated systems score may have diverse outcomes. Thus, there is a need to add prognostic value to the current staging system, which could be achieved with the use of validated biomarkers. Nevertheless, despite numerous studies, no reliable prognostic biomarkers for ccRCC have been identified or used routinely in clinical practice to date.

As DNA methylation is a crucial factor for cancer formation, it rapidly gained clinical attention as a biomarker for diagnosis and prognosis ${ }^{4-6}$. DNA methylation almost exclusively occurs at the C-5 position of cytosines in the sequence context of $5^{\prime}-\mathrm{CpG}-3^{\prime}$ in mammalian cells. As genome-wide technologies continue to develop, such as the development of the Infinium HumanMethylation27 array and HumanMethylation450 array, the understanding of $\mathrm{CpG}$ methylation associated with human cancers including RCC continues to rapidly improve ${ }^{7-12}$.

Here we develop and validate a practical and reliable classifier based on genome-wide CpG methylation profiling that improves risk stratification for patients with ccRCC. Moreover, we use the Cancer Genome Atlas (TCGA) data set to validate our prognostic classifier, investigate the relationship between $\mathrm{CpG}$ methylation and gene expression, and analyse the gene interaction network.

\section{Results}

Identifying candidate CpGs based on genome-wide profiling. We analysed 46 paired ccRCC and adjacent normal tissues by CpG methylation microarray (Infinium HumanMethylation450 array) in the discovery set (Supplementary Table 1) and looked for differential methylation in ccRCC tumours and normal tissue at CpG sites across the genome (Fig. 1). The volcano plot (Fig. 2a) showed that the $\log _{2}$ fold change of $102 \mathrm{CpG}$ sites was more than 2.5 for 46 pairs of tumour and adjacent normal tissue, based on the genome- wide analysis of $\mathrm{CpG}$ methylation ( $t$-test, all $P<10^{-9}$; false discovery rate $<10^{-8}$; Supplementary Data 1). The $102 \mathrm{CpGs}$ identified in univariate analysis were entered into a multivariate logistic regression model (the least absolute shrinkage and selection operator (LASSO)) and 18 had non-zero coefficients (Fig. 2b,c).

Constructing and validating the CpG-based classifier. We then carried out pyrosequencing to quantify the methylation value of these $18 \mathrm{CpG}$ sites by using formalin-fixed, paraffin-embedded (FFPE) specimens from the Sun Yat-sen University (SYSU) set of 168 ccRCC patients. Supplementary Table 3 shows univariate Cox regression analysis of overall survival based on each of the $18 \mathrm{CpGs}$ in the SYSU set $(P=0.49-0.001)$. We used a multivariate LASSO Cox regression model to build a CpG-based prognostic classifier, which included 5 of the 18 CpGs: cg00396667, cg18815943, cg03890877, cg07611000 and cg14391855 (Fig. 2d and Supplementary Fig. 1). These five CpG sites were in the regions of genes PITX1, FOXE3, TWF2, EHBP1L1 and RIN1, respectively. Using the LASSO Cox regression models, we also calculated a risk score for each patient based on individualized values of methylation for the five genes: risk score $=(0.0066 \times$ PITX1 $)+(0.0034 \times$ FOXE3 $)-(0.027 \times$ TWF2 $)$ $-(0.018 \times$ EHBP1L1 $)-(0.03 \times$ RIN 1$)$. When we assessed the distribution of risk scores for the five-CpG-based classifier and survival status, patients with lower risk scores generally had better survival than those with higher risk scores (Fig. 3a, left panel). Patients in the SYSU set were divided into high-risk or low-risk groups, using the median risk score $(-0.1)$ as the cutoff. Compared with patients in low-risk group, patients in the highrisk group had shorter overall survival (hazard ratio $=4.27,95 \%$ confidence interval $=2.18-8.37, \log$-rank test $P=3.9 \times 10^{-6}$; Fig. 3a, right panel).

To estimate the reproducibility and validity of the five-CpGbased classifier, we performed international validation using data sets comprising ccRCC patients from a site in the United States (University of Texas Southwestern Medical Center at Dallas, UTSW set, 243 cases) and multiple clinical centres in China (MCHC set, 284 cases). Furthermore, we used the external data set, TCGA data set (298 cases), to validate our five-CpG-based classifier (Fig. 1 and Table 1). Methylation value of the five CpG

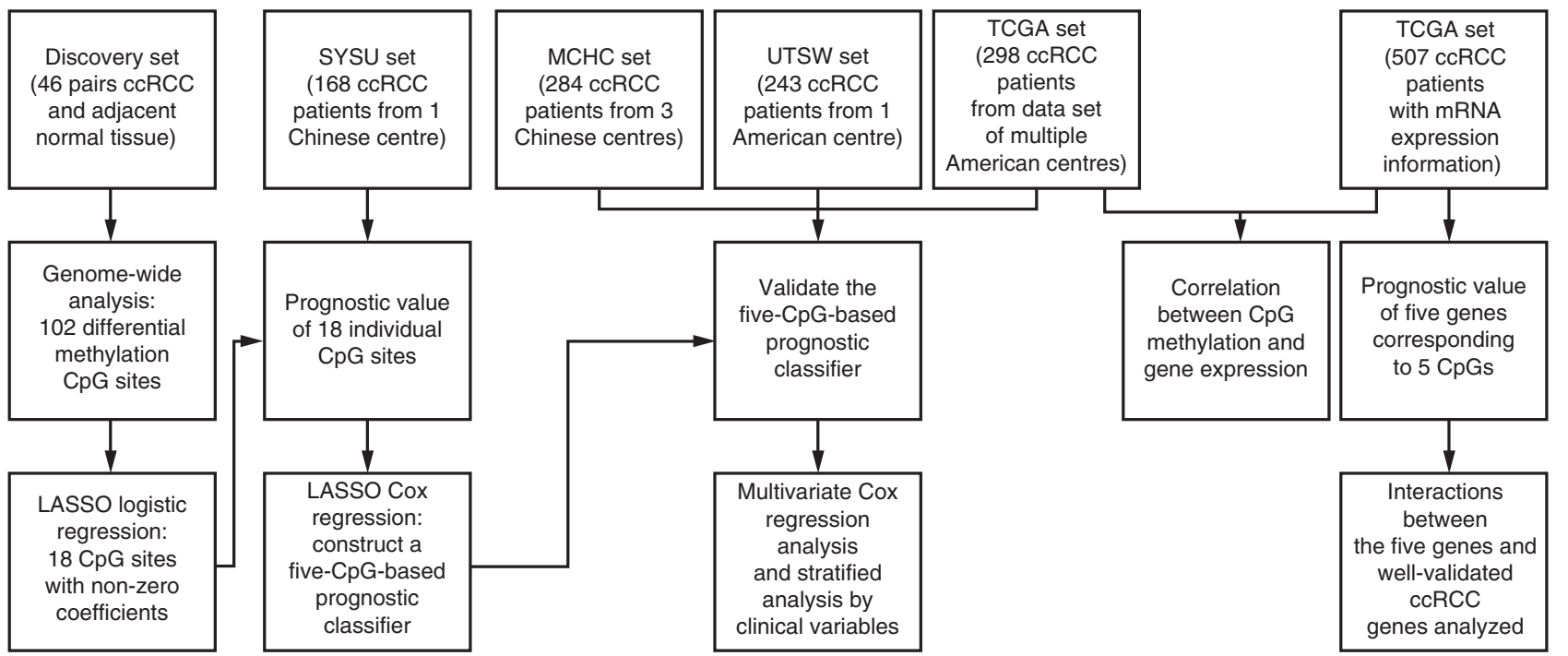

Figure 1 | Flow chart indicating study design. We identified candidate $\mathrm{CpGs}$ sites from 46 paired ccRCC and adjacent normal tissues by $\mathrm{CpG}$ methylation microarray in the discovery set. We then used a multivariate LASSO Cox regression model to build a CpG-based prognostic classifier in SYSU set. Furthermore, the five-CpG-based classifier was validated in MCHC, UTSW and TCGA data sets. Relationship between CpG methylation, gene expression and patient prognosis were also analysed in the TCGA set. 


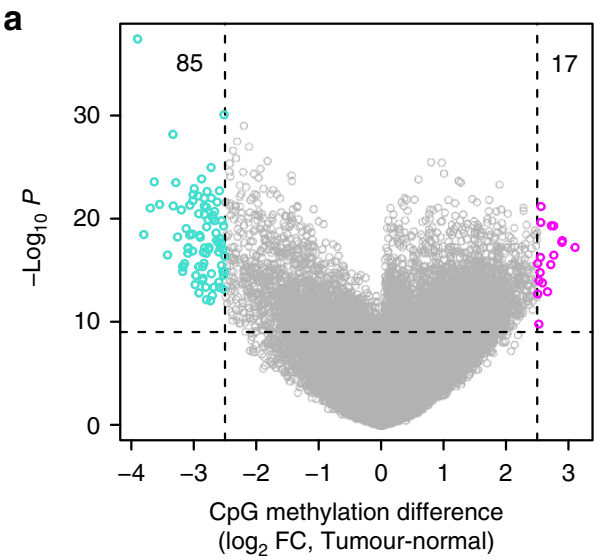

C

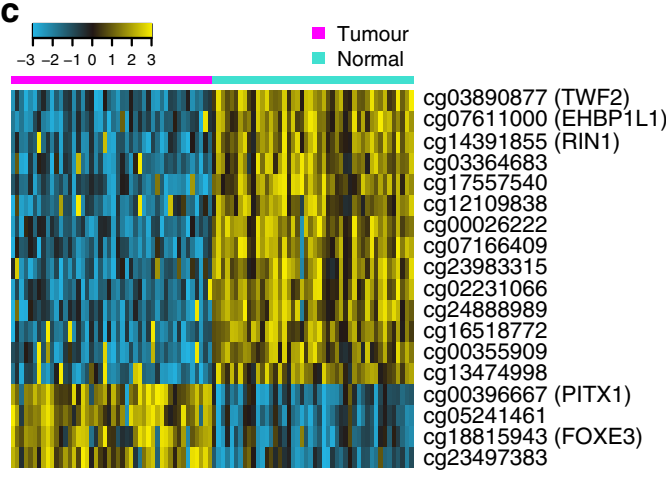

b

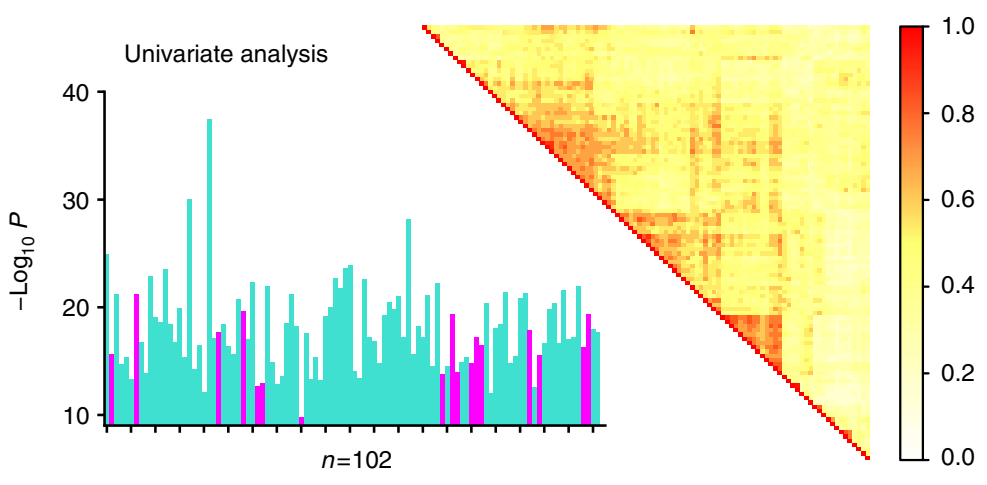

d
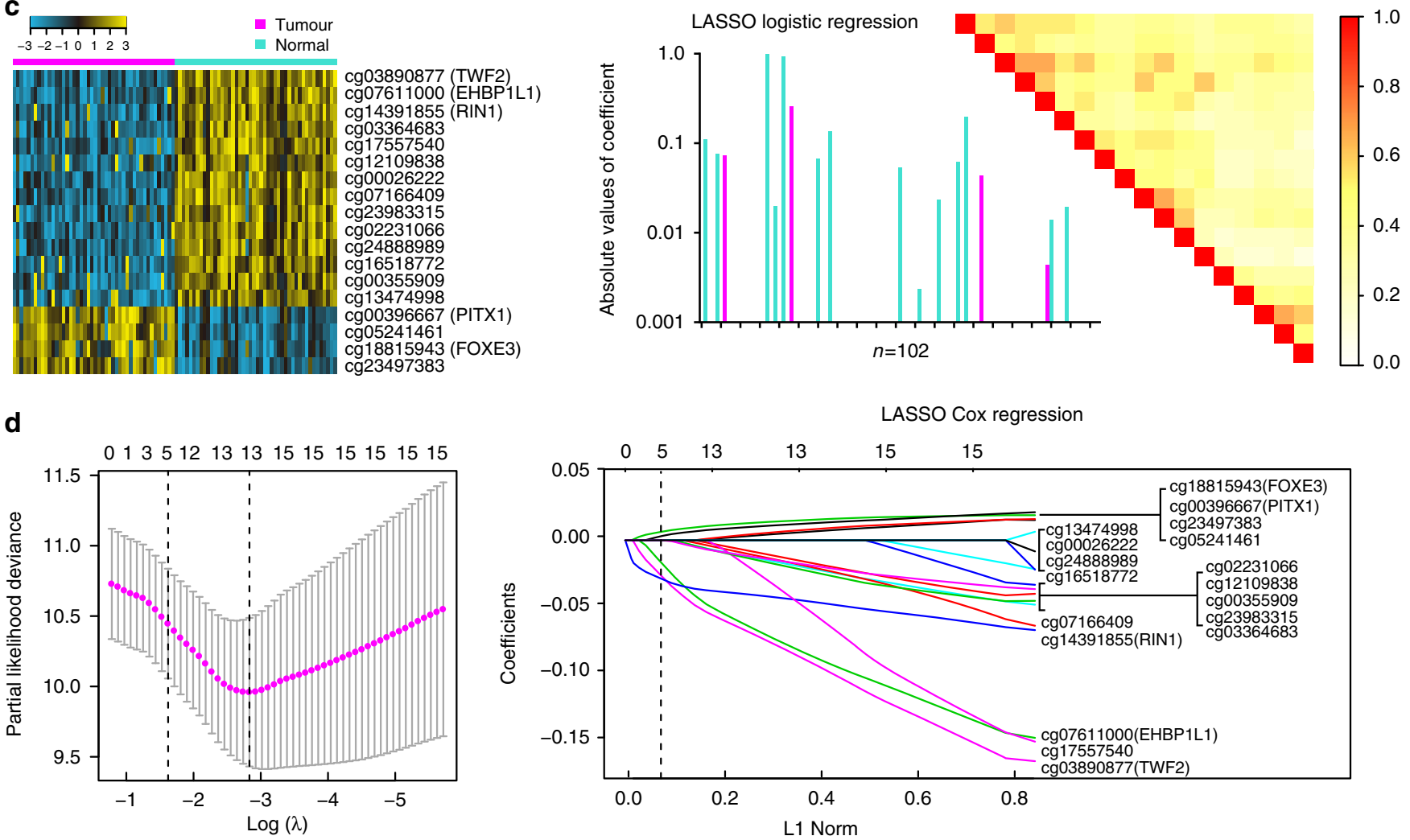

Figure 2 | Construction of the five-CpG-based classifier. (a) One hundred and two CpG sites selected by univariate analysis. Volcano plot showing a comparison of $\mathrm{CpG}$ methylation for ccRCC tumour tissues versus adjacent normal tissues ( $n=46$, HumanMethylation450 platform). This plot depicts the biological significance ( $\log _{2}$ fold change $(F C)$ ) on the $X$ axis and the statistical significance $\left(-\log _{10} P\right)$ on the $Y$ axis. $\log _{2}$ FC $>2 \cdot 5$ for 102 CpGs; the methylation level of $17 \mathrm{CpGs}$ is higher in tumour in comparison with normal tissue (magenta) and lower in $85 \mathrm{CpGs}$ (turquoise). (b) Eighteen CpG sites selected by LASSO logistic regression analysis. Histogram of the univariate $t$-test $P$-values is shown, in the upper left panel, as $-\log _{10} P$ for all $102 \mathrm{CpGs}$. A matrix representing the pairwise correlation ( $r^{2}$, Spearman's correlation) between the $\mathrm{CpGs}$ is displayed in the upper right panel. The lower left panel shows a histogram of the absolute values of the coefficients for all $102 \mathrm{CpGs}$, of which 18 had non-zero coefficients by LASSO logistic regression analysis. The correlation structure between the $18 \mathrm{CpGs}$ with non-zero coefficients is shown in the lower right panel, demonstrating reduced multicollinearity. (c) Heatmap showing methylation of the $18 \mathrm{CpGs}$ in ccRCC tumour tissue (46 samples) and adjacent normal tissue (46 samples). (d) Five CpG sites selected by LASSO Cox regression analysis. Left panel: the two dotted vertical lines are drawn at the optimal values by minimum criteria (right) and $1-$ s.e. criteria (left). Details are provided in Methods. Right panel: LASSO coefficient profiles of the $18 \mathrm{CpGs}$. A vertical line is drawn at the optimal value by 1 - s.e. criteria and results in five non-zero coefficients. Five CpGs-cg00396667 (PITX1), cg18815943 (FOXE3), cg03890877 (TWF2), cg07611000 (EHBP1L1) and cg14391855 (RIN1)-with coefficients $0.0066,0.0034,-0.027,-0.018$ and -0.03 , respectively, were selected in the LASSO Cox regression model.

sites is shown for each set in Supplementary Fig. 2. The risk score for each patient in the sets was calculated with the same formula used in the SYSU set, patients with lower risk scores generally had better survival than those with higher risk scores (Fig. 3b-d, left panel). Patients in these three sets were classified into high-risk and low-risk groups with the same cutoff used in the SYSU set $(-0.1)$. Patients in the high-risk groups had shorter overall survival than those in the low-risk groups in all three sets (hazard ratio $=2.96-4.82, \quad$ log-rank test $P=1.4 \times 10^{-6}-2.2 \times 10^{-9}$; Fig. 3b-d (right panel) and Supplementary Table 4). After 

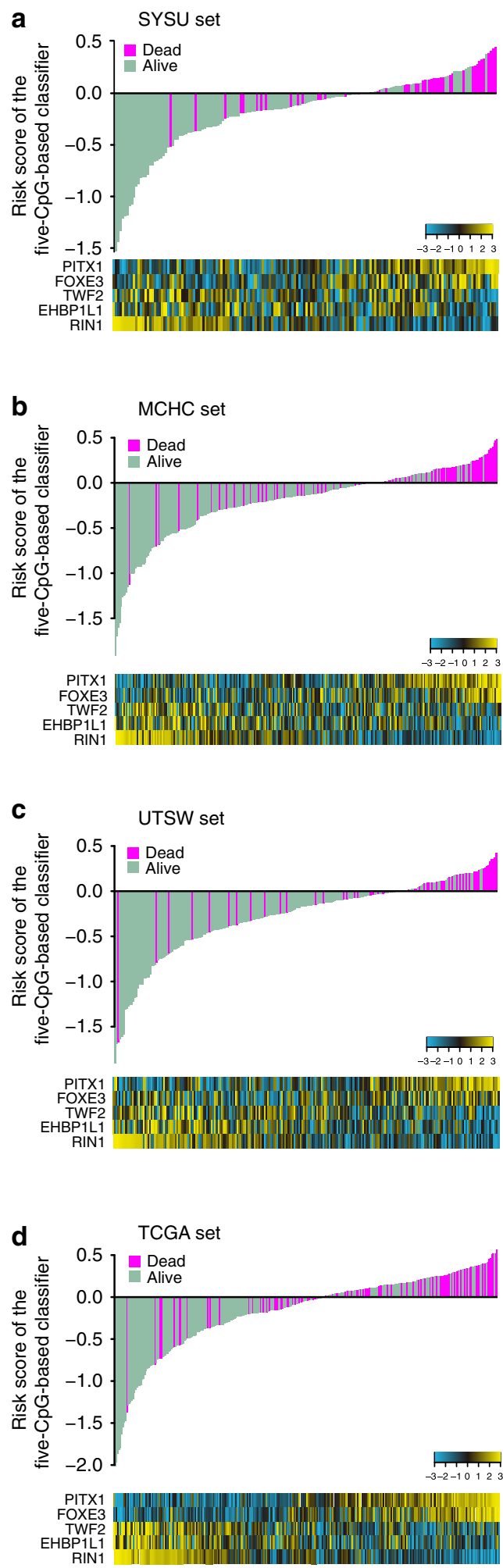
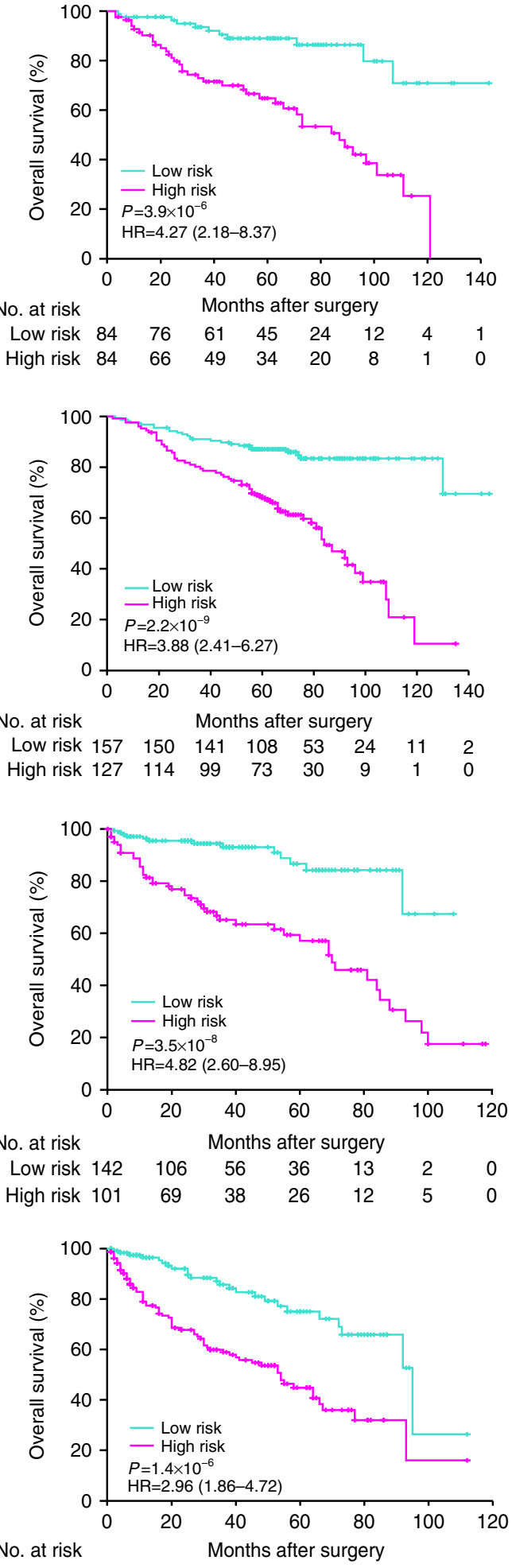

o. at risk

$\begin{array}{ccccccc}\text { Low risk 138 } & 82 & 53 & 31 & 14 & 1 & 0 \\ \text { High risk } 160 & 90 & 56 & 27 & 7 & 1 & 0\end{array}$

Figure 3 | Risk score calculated by the five-CpG-based classifier and Kaplan-Meier survival in the four different sets. (a) SYSU set, (b) MCHC set, (c) UTSW set and (d) TCGA set. Upper left panel: risk-score distribution of the five-CpG-based classifier and patient survival status. Lower left panel: heatmap showing methylation of the five $\mathrm{CpGs}$ in the patients. Right panel: Kaplan-Meier survival analysis for the patients. The patients were divided into low-risk and high-risk groups using the median cutoff value of the classifier risk score $(-0.1)$. $P$-values were calculated using the log-rank test. $\mathrm{HR}$, hazard ratio. 
Table 1 | Baseline characteristics of patients by the five-CpG-based classifier assessment set.

\begin{tabular}{|c|c|c|c|c|c|c|c|c|c|c|c|c|}
\hline \multirow[t]{2}{*}{ Characteristic } & \multicolumn{3}{|c|}{ SYSU set $(n=168)$} & \multicolumn{3}{|c|}{ MCHC set $(n=284)$} & \multicolumn{3}{|c|}{ UTSW set $(n=243)$} & \multicolumn{3}{|c|}{ TCGA set $(n=298)$} \\
\hline & $\begin{array}{c}\text { No.of } \\
\text { patients }\end{array}$ & $\begin{array}{l}\text { Low risk } \\
\text { (\%) }\end{array}$ & $\begin{array}{c}\text { High risk } \\
(\%)\end{array}$ & $\begin{array}{c}\text { No.of } \\
\text { patients }\end{array}$ & $\begin{array}{c}\text { Low risk } \\
(\%)\end{array}$ & $\begin{array}{l}\text { High risk } \\
\text { (\%) }\end{array}$ & $\begin{array}{c}\text { No.of } \\
\text { patients }\end{array}$ & $\begin{array}{l}\text { Low risk } \\
\text { (\%) }\end{array}$ & $\begin{array}{c}\text { High risk } \\
(\%)\end{array}$ & $\begin{array}{c}\text { No.of } \\
\text { patients }\end{array}$ & $\begin{array}{c}\text { Low risk } \\
(\%)\end{array}$ & $\begin{array}{l}\text { High risk } \\
\text { (\%) }\end{array}$ \\
\hline \multicolumn{13}{|l|}{ Age (years) } \\
\hline$<60$ & 107 & $51(48 \%)$ & $56(52 \%)$ & 178 & $104(58 \%)$ & $74(42 \%)$ & 128 & $82(64 \%)$ & $46(36 \%)$ & 129 & $71(55 \%)$ & $58(45 \%)$ \\
\hline$\geq 60$ & 61 & $33(54 \%)$ & $28(46 \%)$ & 106 & $53(50 \%)$ & $53(50 \%)$ & 115 & $60(52 \%)$ & $55(48 \%)$ & 169 & $67(40 \%)$ & $102(60 \%)$ \\
\hline \multicolumn{13}{|l|}{ Sex } \\
\hline Male & 113 & $55(49 \%)$ & $58(51 \%)$ & 190 & $109(57 \%)$ & $81(43 \%)$ & 151 & $87(58 \%)$ & $64(42 \%)$ & 193 & $71(37 \%)$ & $122(63 \%)$ \\
\hline Female & 55 & $29(53 \%)$ & $26(47 \%)$ & 94 & $48(51 \%)$ & $46(49 \%)$ & 92 & $55(60 \%)$ & $37(40 \%)$ & 105 & $67(64 \%)$ & $38(36 \%)$ \\
\hline \multicolumn{13}{|l|}{ Race } \\
\hline Asian & 168 & $84(50 \%)$ & $84(50 \%)$ & 284 & $157(55 \%)$ & $127(45 \%)$ & 4 & $1(25 \%)$ & $3(75 \%)$ & 1 & $0(0 \%)$ & $1(100 \%)$ \\
\hline White & 0 & & & 0 & & & 183 & $104(57 \%)$ & $79(43 \%)$ & 264 & $120(45 \%)$ & $144(55 \%)$ \\
\hline Black & 0 & & & 0 & & & 36 & $23(64 \%)$ & $13(36 \%)$ & 30 & $18(60 \%)$ & $12(40 \%)$ \\
\hline $\begin{array}{l}\text { Not } \\
\text { available }\end{array}$ & 0 & & & 0 & & & 20 & $14(70 \%)$ & $6(30 \%)$ & 3 & $0(0 \%)$ & $3(100 \%)$ \\
\hline \multicolumn{13}{|l|}{ Grade } \\
\hline G1 & 8 & $6(75 \%)$ & $2(25 \%)$ & 21 & $15(71 \%)$ & $6(29 \%)$ & 10 & $8(80 \%)$ & $2(20 \%)$ & 6 & $6(100 \%)$ & $0(0 \%)$ \\
\hline $\mathrm{G} 2$ & 87 & $42(48 \%)$ & $45(52 \%)$ & 134 & $80(60 \%)$ & $54(40 \%)$ & 128 & $84(66 \%)$ & $44(34 \%)$ & 123 & $75(61 \%)$ & $48(39 \%)$ \\
\hline G3 & 51 & $25(49 \%)$ & $26(51 \%)$ & 88 & $45(51 \%)$ & $43(49 \%)$ & 77 & $38(49 \%)$ & $39(51 \%)$ & 120 & $50(42 \%)$ & $70(58 \%)$ \\
\hline G4 & 22 & $11(50 \%)$ & $11(50 \%)$ & 41 & $17(41 \%)$ & $24(59 \%)$ & 28 & $12(43 \%)$ & $16(57 \%)$ & 49 & $7(14 \%)$ & $42(86 \%)$ \\
\hline \multicolumn{13}{|l|}{ Tumour size } \\
\hline$<5 \mathrm{~cm}$ & 60 & $33(55 \%)$ & $27(45 \%)$ & 140 & $76(54 \%)$ & $64(46 \%)$ & 136 & $93(68 \%)$ & $43(32 \%)$ & 119 & $76(64 \%)$ & $43(36 \%)$ \\
\hline$\geq 5 \mathrm{~cm}$ & 108 & $51(47 \%)$ & $57(53 \%)$ & 144 & $81(56 \%)$ & $63(44 \%)$ & 107 & $49(46 \%)$ & $58(54 \%)$ & 178 & $62(35 \%)$ & $116(65 \%)$ \\
\hline $\begin{array}{l}\text { Not } \\
\text { available }\end{array}$ & 0 & & & 0 & & & 0 & & & 1 & $0(0 \%)$ & $1(100 \%)$ \\
\hline \multicolumn{13}{|l|}{ Tumour necrosis } \\
\hline Absent & 104 & $56(54 \%)$ & $48(46 \%)$ & 189 & $102(54 \%)$ & $87(46 \%)$ & 164 & $103(63 \%)$ & $61(37 \%)$ & 138 & $71(51 \%)$ & 67 (49\%) \\
\hline Present & 64 & $28(44 \%)$ & $36(56 \%)$ & 95 & $55(58 \%)$ & $40(42 \%)$ & 70 & $32(46 \%)$ & $38(54 \%)$ & 160 & $67(42 \%)$ & $93(58 \%)$ \\
\hline $\begin{array}{l}\text { Not } \\
\text { available }\end{array}$ & 0 & & & 0 & & & 9 & $7(78 \%)$ & $2(22 \%)$ & 0 & & \\
\hline \multicolumn{13}{|l|}{$p T$} \\
\hline $\mathrm{T} 1$ & 97 & $49(51 \%)$ & $48(49 \%)$ & 180 & $101(56 \%)$ & 79 (44\%) & 156 & 107 (69\%) & $49(31 \%)$ & 145 & $95(66 \%)$ & $50(34 \%)$ \\
\hline $\mathrm{T} 2$ & 30 & $15(50 \%)$ & $15(50 \%)$ & 54 & $27(50 \%)$ & $27(50 \%)$ & 30 & $10(33 \%)$ & $20(67 \%)$ & 38 & $18(47 \%)$ & $20(53 \%)$ \\
\hline T3 & 37 & $17(46 \%)$ & $20(54 \%)$ & 46 & $27(59 \%)$ & $19(41 \%)$ & 52 & $24(46 \%)$ & $28(54 \%)$ & 107 & $23(21 \%)$ & $84(79 \%)$ \\
\hline $\mathrm{T} 4$ & 4 & $3(75 \%)$ & $1(25 \%)$ & 4 & $2(50 \%)$ & $2(50 \%)$ & 5 & $1(20 \%)$ & $4(80 \%)$ & 8 & $2(25 \%)$ & $6(75 \%)$ \\
\hline \multicolumn{13}{|l|}{$p N$} \\
\hline NO & 152 & $78(51 \%)$ & 74 (49\%) & 267 & $151(57 \%)$ & $116(43 \%)$ & 226 & $134(59 \%)$ & $92(41 \%)$ & 129 & $62(48 \%)$ & $67(52 \%)$ \\
\hline N1 & 16 & $6(37 \%)$ & $10(63 \%)$ & 17 & $6(35 \%)$ & $11(65 \%)$ & 17 & $8(47 \%)$ & $9(53 \%)$ & 8 & $1(12 \%)$ & $7(88 \%)$ \\
\hline NX & 0 & & & 0 & & & 0 & & & 161 & $75(47 \%)$ & $86(53 \%)$ \\
\hline \multicolumn{13}{|l|}{ M } \\
\hline MO & 163 & $83(51 \%)$ & $80(49 \%)$ & 274 & $150(55 \%)$ & $124(45 \%)$ & 221 & $136(62 \%)$ & $85(38 \%)$ & 244 & 125 (51\%) & 119 (49\%) \\
\hline M1 & 5 & $1(20 \%)$ & $4(80 \%)$ & 10 & $7(70 \%)$ & $3(30 \%)$ & 22 & $6(27 \%)$ & $16(73 \%)$ & 54 & $13(24 \%)$ & $41(76 \%)$ \\
\hline \multicolumn{13}{|l|}{ Stage (clinical) } \\
\hline Stage I & 91 & 45 (49\%) & $46(51 \%)$ & 171 & $96(56 \%)$ & 75 (44\%) & 155 & 107 (69\%) & $48(31 \%)$ & 141 & $95(67 \%)$ & $46(33 \%)$ \\
\hline Stage II & 27 & $15(56 \%)$ & $12(44 \%)$ & 48 & $24(50 \%)$ & $24(50 \%)$ & 25 & $9(36 \%)$ & $16(64 \%)$ & 28 & $15(54 \%)$ & $13(46 \%)$ \\
\hline Stage III & 36 & $17(47 \%)$ & $19(53 \%)$ & 43 & $28(65 \%)$ & $15(35 \%)$ & 39 & $20(51 \%)$ & 19 (49\%) & 73 & $15(20 \%)$ & $58(80 \%)$ \\
\hline Stage IV & 14 & $7(50 \%)$ & $7(50 \%)$ & 22 & 9 (41\%) & $13(59 \%)$ & 24 & $6(25 \%)$ & $18(75 \%)$ & 56 & $13(23 \%)$ & $43(77 \%)$ \\
\hline
\end{tabular}

MCHC, multiple clinical centres in China; SYSU, Sun Yat-sen University; TCGA, The Cancer Genome Atlas; UTSW, University of Texas Southwestern Medical Center at Dallas.

adjusting for standard clinical prognostic factors (age, TNM stage, Fuhrman grade and necrosis status), the five-CpG-based classifier remained an independent prognostic factor in the SYSU set and the three other patient sets (Table 2, all $P<0.05$ ).

Stratification analysis of the five-CpG-based classifier. Survival analysis was further performed with regard to the five-CpG-based classifier in subsets of patients with different clinical variables. When stratified by clinical variables (sex, age, race, Fuhrman grade, tumour size and necrosis status), the five-CpG-based classifier was still a clinically and statistically significant prognostic model (Fig. 4a, Supplementary Fig. 3 and Supplementary Table 5). As shown in Fig. $4 \mathrm{~b}$, the ccRCC patients in the same clinical stage could be successfully separated into the subgroups of better prognosis and poorer prognosis by the five-CpG-based classifier (log-rank test, all $P<0.05$ ).

The SSIGN score (ranging from 0 to 15 ) is one of the clinically integrated systems that was introduced to improve prognostic accuracy in ccRCC (Supplementary Table 6). The Kaplan-Meier curves regarding overall survival for respective SSIGN-score categories are shown in Fig. 5a. The five-CpG-based classifier successfully categorized patients into high-risk and low-risk groups with significant differences of clinical outcome in each of the SSIGN-score categories (log-rank test, all $P<0.05$; Fig. 5b-f). Thus, the five-CpG-based classifier can add prognostic value to both the clinical stage and the SSIGN score.

Impact of intratumour heterogeneity. To determine whether intratumour heterogeneity (ITH) affected risk score and risk stratification based on the five-CpG-based classifier, we assayed methylation value of the five $\mathrm{CpG}$ sites in three different regions within 23 ccRCC tumours. As shown in Supplementary Fig. 5, inter-individual differences in the methylation of the five CpG sites, assessed by averaging all measurements from the same tumour, were significantly higher than measurement differences within individual tumours. ITH had an obviously smaller effect on classifier-based risk scores (coefficient of variation $(\mathrm{CV})$, $10.5 \%$ ) than on the five individual CpGs (CV, 15.2-22.3\%). 
Table 2 | Multivariate Cox regression analysis of the five-CpG-based classifier with overall survival in the four sets.

\begin{tabular}{|c|c|c|c|c|c|c|c|c|}
\hline $\begin{array}{l}\text { Age (younger than } 60 \text { years } \\
\text { versus } 60 \text { years or older) }\end{array}$ & $1.18(0.66-2.11)$ & 0.58 & $2.13(1.36-3.33)$ & 0.001 & $1.76(0.98-3.14)$ & 0.06 & $1.28(0.81-2.02)$ & 0.29 \\
\hline pT $(T 1 / 2$ versus $T 3 / 4)$ & $2.82(1.42-5.56)$ & 0.003 & $1.99(1.20-3.31)$ & 0.008 & $2.39(1.27-4.50)$ & 0.007 & $1.63(1.01-2.63)$ & 0.05 \\
\hline $\mathrm{pN}$ (N0 versus $\mathrm{N} 1$ ) & $3.16(1.37-7.28)$ & 0.007 & $4.59(2.39-8.83)$ & $<0.001$ & $2.01(0.95-4.26)$ & 0.07 & $-^{\star}$ & $-^{\star}$ \\
\hline$M(\mathrm{MO}$ versus $\mathrm{M} 1)$ & $7.41(1.97-27.89)$ & 0.003 & $1.61(0.60-4.27)$ & 0.34 & $3.10(1.46-6.57)$ & 0.003 & $2.77(1.78-4.31)$ & $<0.001$ \\
\hline $\begin{array}{l}\text { Five-CpG-based classifier } \\
\text { (low versus high risk) }\end{array}$ & $4.10(2.05-8.19)$ & $<0.001$ & $3.73(2.28-6.09)$ & $<0.001$ & $3.36(1.78-6.34)$ & $<0.001$ & $1.80(1.11-2.93)$ & 0.02 \\
\hline
\end{tabular}

ITH affected risk stratification in $2(8.7 \%)$ of the 23 tumours, suggesting the 5 -CpG-based classifier is a precise tool (Supplementary Table 7).

CpG methylation and gene expression and patient prognosis. Using the TCGA data set, we analysed whether methylation of the five CpGs was correlated with gene expression, as per Spearman's correlation. We observed that the correlation between methylation value and gene expression by Spearman's correlation test was significantly inverse for TWF2 $\left(P=5.8 \times 10^{-11}\right)$, EHBP1L1 $\left(P=1.9 \times 10^{-6}\right)$ and RIN1 $\left(P=1.2 \times 10^{-30}\right)$, significantly positive for PITX1 $\left(P=4.1 \times 10^{-8}\right)$ and marginally positive for FOXE3 $(P=0.09)$.

Nine hundred and ninety-three patients in the entire cohort were separated into CpG-defined high-risk and low-risk groups using X-tile plots, to generate the optimum cutoff score for methylation of the five CpGs. Kaplan-Meier survival analysis, depicted in Fig. 6a-e (left panel), showed the overall survival of patients in the CpG-defined low-risk group was significantly better than in the high-risk group. In addition, expression of the genes corresponding to the $5 \mathrm{CpGs}$ effectively predicted the clinical outcome of the 507 patients for whom there were messenger RNA expression data in the TCGA data set (Fig. 6a-e, right panel).

Integrating our results with genes linked to RCC. To further evaluate the role of genes corresponding to the five CpGs in relation to well-validated ccRCC susceptibility genes, we used the cBioPortal for Cancer Genomics network to evaluate gene connectivity. As shown in Fig. 6f, PITX1 interacts with EGR1, which is then connected to an immune response network. RIN1 interacts with $R A B 5 A$, which is connected to genes that are involved in cancer cell epithelial-to-mesenchymal transition. TWF2 mainly participates in cancer cell proliferation signalling pathways through interaction with chromogranin B (CHGB). FOXE3 and $E H B P 1 L 1$ showed exceptionally low connectivity in the database.

\section{Discussion}

Integrating multiple biomarkers into a single model would substantially improve prognostic value compared with a single biomarker ${ }^{13}$. As genome-wide technologies have become more sophisticated, so too have molecular prognostic models, which can now integrate mRNA, microRNA, CpG and single-nucleotide polymorphism (SNP) data ${ }^{7,14-19}$. However, early studies with integrated models had several notable limitations. (1) There was a lack of information (such as risk score formulas or biomarker coefficients) on how to integrate multiple biomarkers into one model, which restricted wide use of these models in the clinic. (2) Some models incorporated too many biomarkers, making it nearly impossible to apply them in clinical practice. (3) Inappropriate statistical methods were used to mine microarray data. More specifically, in microarray analysis, the number of covariates is usually close to or larger than the number of observations. The Cox proportional hazards regression analysis, which is the most popular approach for modelling covariate information for survival times, is unsuitable for highdimensional microarray data when the sample-size-to-variables ratio is too low (such as $<10: 1)^{20,21}$. The LASSO model used in our study is one of the statistical methods that can eliminate this limitation ${ }^{22-24}$. (4) Models were developed based on analysis of fresh-frozen specimens, limiting immediate clinical application in a broad community setting. (5) Models were not validated in multiple independent cohorts. Thus, none of the integrated prognostic models developed using genome-wide, microarraybased analysis are being used in clinical practice. In this study, we developed a practical CpG-methylation-based assay that can be used with FFPE material to identify prognostic CpG information and demonstrated how this information can be integrated into a prognostic model that is feasible to use in the clinic.

ITH can impair the precise molecular analysis of tumours, because biomarker expression can vary across different tumour regions ${ }^{25}$. Some prognostic biomarkers could not be validated in previous reports and one possible cause was large intra-sample variability in gene expression ${ }^{26}$. However, two recent studies showed ITH, although present at the level of individual gene expression, did not preclude precise microarray-based predictions of clinical outcome in ccRCC or breast cancer ${ }^{26,27}$. Compared with a single prognostic biomarker, our integrated prognostic models based on microarray profiling not only have higher prognostic accuracy but also are less influenced by ITH.

Several studies have analysed gene expression profiles in RCC and examined their potential clinical relevance ${ }^{28-31}$. These signatures contained large numbers of genes that were detected by microarray or reverse transcriptase-PCR and, consequently, these signatures had limited use in clinical practice. In this study, we identified methylation level of five highly prognostic $\mathrm{CpG}$ sites by pyrosequencing from the FFPE material. Given the fewer number of markers, our classifier is both more feasible and cheaper compared with the prognostic signatures proposed in previous studies. The five-CpG-based classifier can accurately distinguish between patients with ccRCC, with substantially 
a

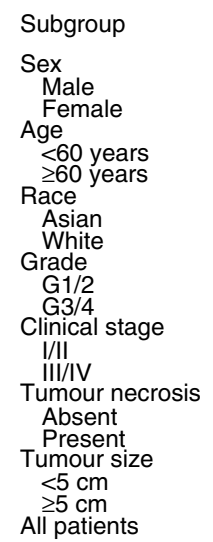

$\mathrm{HR}(95 \% \mathrm{Cl})$

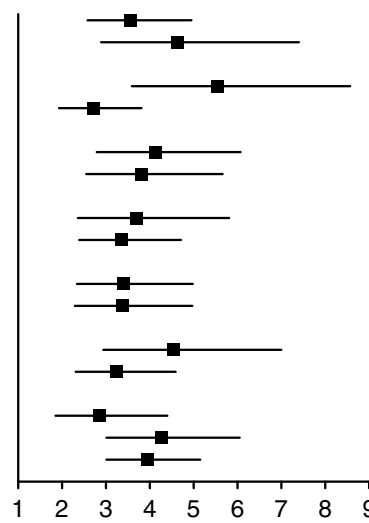

Endpoint

$3.57(2.58-4.95)$

$4.63(2.89-7.40)$

$5.55(3.59-8.57)$

$2.71(1.93-3.81)$

$4.12(2.79-6.07)$

$3.80(2.55-5.66)$

$3.70(2.36-5.81)$

$3.35(2.39-4.71)$

$3.41(2.34-4.98)$

$3.38(2.29-4.97)$

$4.54(2.94-7.00)$

$3.25(2.31-4.59)$

2.85 (1.85-4.40)

$4.27(3.01-6.05)$
$P$-value

$<0.001$

$<0.001$

$<0.001$

$<0.001$

$<0.001$

$<0.001$

$<0.001$

$<0.001$

$<0.001$

$<0.001$

$<0.001$

$<0.001$

$<0.001$

$<0.001$

b

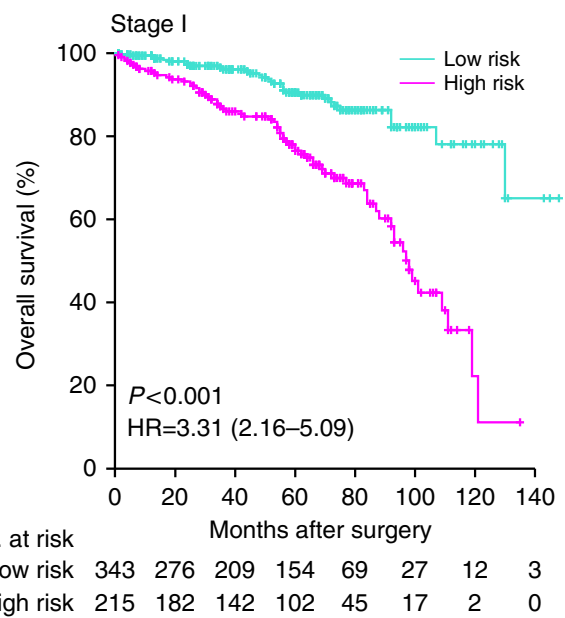

Stage II
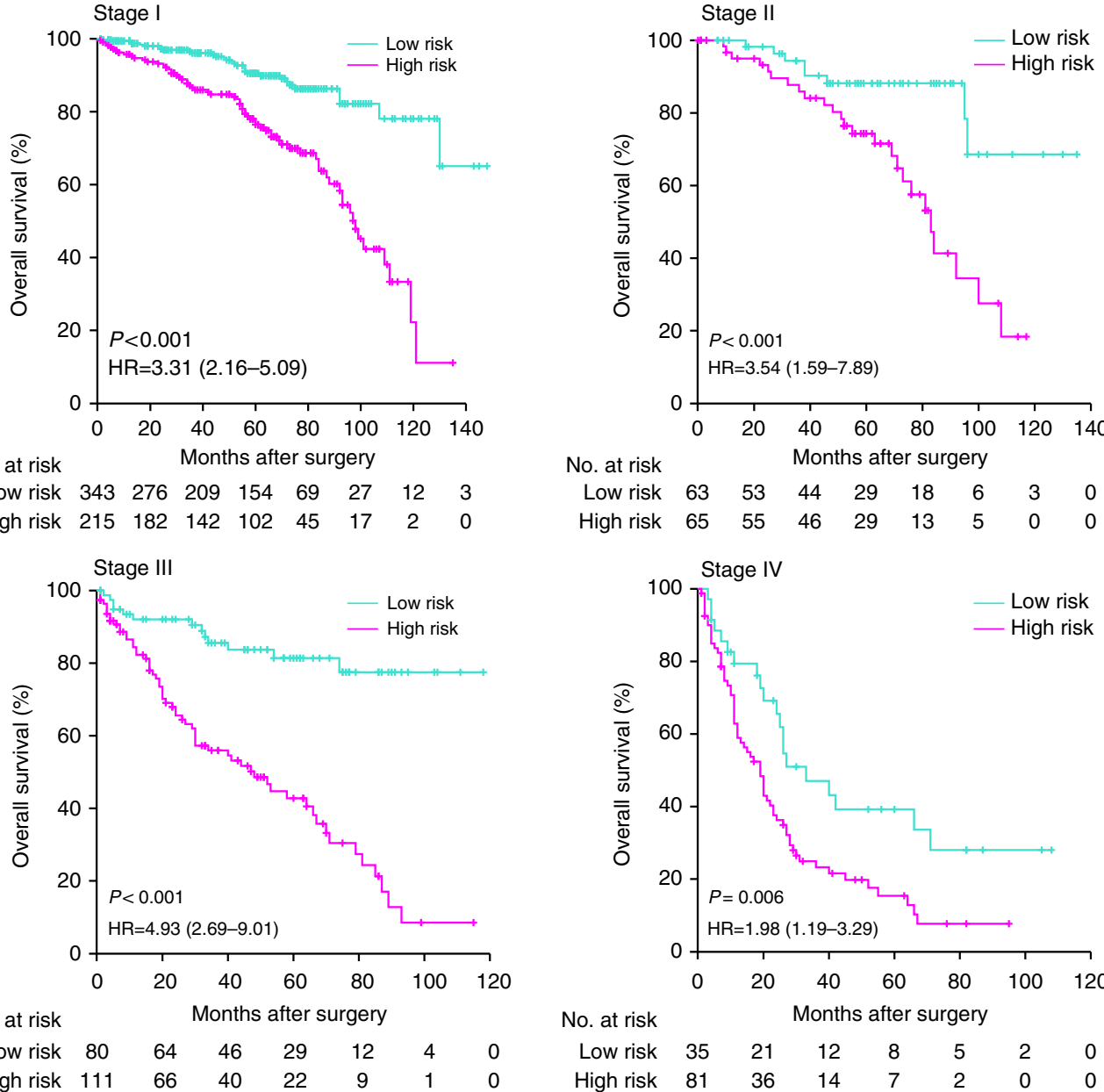

Figure 4 | Stratification analysis of the five-CpG-based classifier. (a) Hazard ratio (HR) of overall mortality for all 993 patients with ccRCC according to the five-CpG-based classifier in different subgroups stratified by clinical parameters. (b) Kaplan-Meier survival analysis of the five-CpG-based classifier in subsets of different clinical stage patients with ccRCC (log-rank test).

different clinical outcomes, even after adjustment for standard clinical prognostic factors, such as age, TNM stage, Fuhrman grade and necrosis status. We further performed international validation using data sets comprising patients from a site in the United States and MCHC, as well as patients in TCGA data set, who were also from multiple centres in the United States. The prognostic accuracy of the five-CpG-based classifier was similar in the three validation sets. The classifier was reproducible regardless of clinical centre, country or race and it can provide prognostic value that complements the clinical stage and the SSIGN score.

Five genes corresponded to the five CpGs identified in our study: FOXE3, PITX1, RIN1, TWF2 and EHBP1L1. DNA methylation of FOXE3 has been reported and validated as a diagnostic biomarker for paediatric acute lymphoblastic leukemia $^{32}$. Hypermethylation of PITX1 and RIN1 has been described in human salivary gland adenoid cystic carcinoma and breast cancer, respectively ${ }^{33,34}$. TWF2 has been implicated in 
a

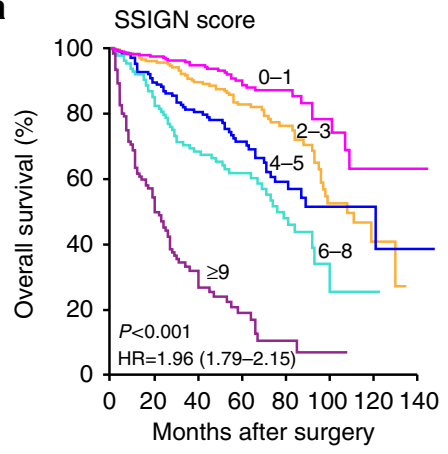

d

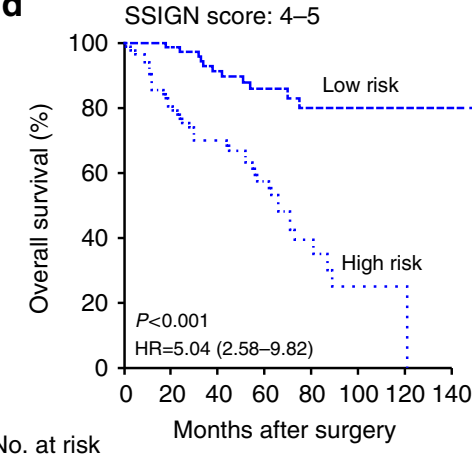

$\begin{array}{llllllll}\text { Low risk } 93 & 74 & 56 & 36 & 19 & 8 & 3 & 1\end{array}$

High risk $89 \begin{array}{llllllll}84 & 47 & 28 & 9 & 4 & 1 & 0\end{array}$ b

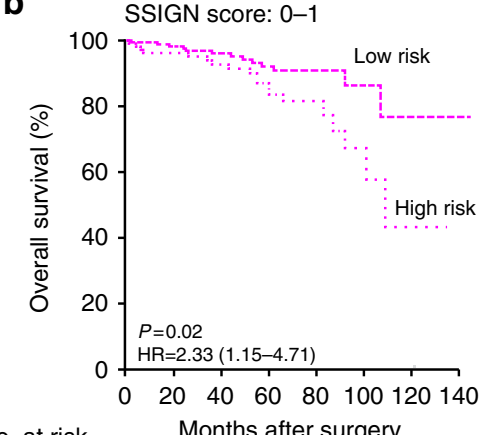

No. at risk

Low risk $182154 \begin{array}{lllllll}108 & 78 & 34 & 13 & 4 & 2\end{array}$

$\begin{array}{lllllllll}\text { High risk } & 106 & 93 & 72 & 50 & 22 & 8 & 1 & 0\end{array}$

e

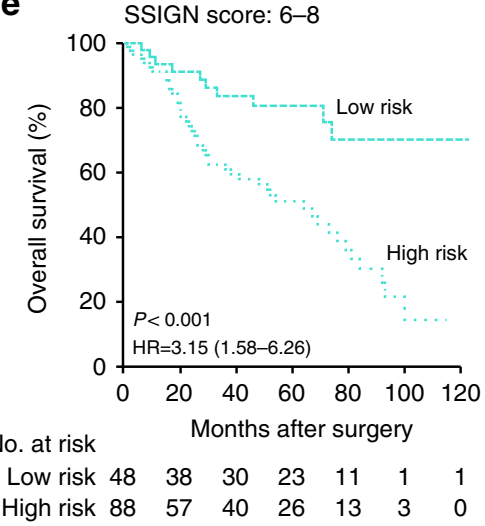

C

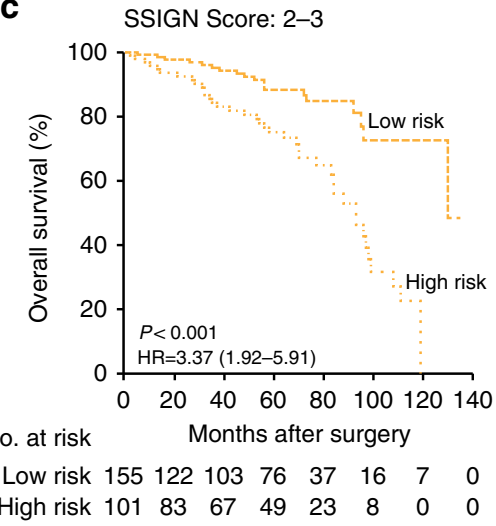

f

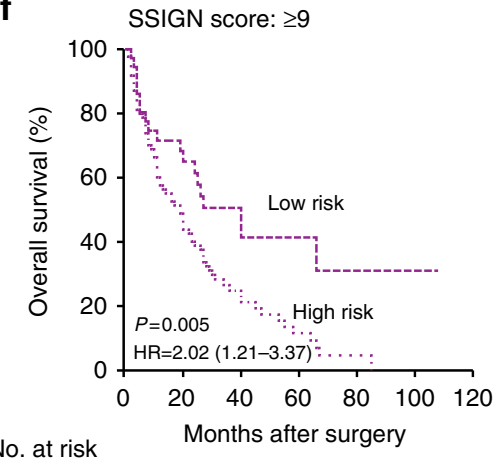

Low risk

$\begin{array}{llllllll}36 & 21 & 11 & 5 & 3 & 1 & 0\end{array}$

Figure 5 | Analysis of the five-CpG-based classifier in subsets of different SSIGN-score categories. (a)The Kaplan-Meier curves regarding overall survival for respective SSIGN-score categories. (b-f) Kaplan-Meier survival analysis of the five-CpG-based classifier in subsets of different SSIGN-score categories (log-rank test). HR, hazard ratio.

neurite outgrowth ${ }^{35}$. However, the function of EHBP1L1 remains unknown. Our pathway analysis results showed that these genes may play diverse roles in regulating ccRCC progression, including tumour immune response, cancer cell proliferation and epithelial-to-mesenchymal transition. Notably, these genes are all distributed at the periphery of the signalling network, in contrast to central network markers such as PTEN and TP53. This finding is similar to recent studies showing that epigenetic marker drift occurs preferentially in genes that occupy peripheral network positions of exceptionally low connectivity $7,36,37$.

In conclusion, the present study suggests the newly developed five-CpG-based classifier is a practical and powerful prognostic tool for ccRCC, which can provide prognostic value that complements the current staging system of ccRCC and will facilitate patient counselling, tailoring of follow-up protocols and selection for appropriate adjuvant trial designs.

\section{Methods}

Patients. In this study, we used 695 FFPE tissue samples from 695 patients who underwent resection of a ccRCC. The SYSU set included 168 patients from the First Affiliated Hospital and Cancer Center of SYSU (Guangdong, Southeast China) treated between 2001 and 2009. The MCHC set included 284 patients treated between 2001 and 2009 at three hospitals across different regions of China: First Affiliated Hospital of Xi'an Jiaotong University (Shaanxi, Northwest China), Affiliated Yantai Yuhuangding Hospital of Qingdao University Medical College (Shandong, Northeast China) and Affiliated Hospital of Kunming University of Science and Technology (Yunnan, Southwest China) between 2001 and 2009. Another 243 patients from the University of Texas Southwestern Medical Center at Dallas (TX, USA) treated between 2004 and 2011 comprised the UTSW set. The TNM 2009 staging system was used to classify ccRCC patients. The grading system used in the study was based on the Fuhrman four grade. Clinical baseline data were obtained through medical record review. Patients with sporadic, unilateral ccRCC and with clinicopathological characteristics and follow-up information available were included. In addition, to generate $\mathrm{CpG}$ methylation expression profiles we obtained, as a discovery set, a panel of 46 fresh-frozen tumour samples with paired adjacent normal tissue from patients with ccRCC treated between 2011 and 2013 at the First Affiliated Hospital of SYSU. Consent was obtained for all subjects and the protocols approved by the respective Institutional Review Board of each institution.

Infinium methylation assay microarrays. In the discovery set, we used the HumanMethylation450 BeadChip (Illumina, San Diego, CA, USA) for genomewide assessment of methylation at $\mathrm{CpG}$ sites ${ }^{38}$. Genomic DNA was extracted from 46 paired ccRCC tumour and adjacent normal tissues with the QIAamp DNA mini kit (Qiagen, Valencia, CA, USA) following the manufacturer's recommendations. All DNA samples were assessed for integrity, quantity and purity by electrophoresis in a $1.3 \%$ agarose gel, PicoGreen quantification and NanoDrop measurements, respectively. The samples that passed quality control were processed with Infinium HumanMethylation450 BeadChip Kits (Illumina) according to the manufacturer's recommendations, through automated processes in the Genomic and Microarray Core, University of Texas Southwestern Medical Center. Arrays were imaged with BeadArray Reader using standard Illumina scanner settings. The signal data were extracted and processed using RnBeads ${ }^{39}$ version 0.99.12 in the R software 3.0.3. We considered a methylation $\beta$-value to be unreliable if its corresponding detection $P$-value was not below the threshold $T=0.05$. Both sites and samples were filtered using a greedy approach. BMIQ normalization methods and the background subtraction 'methylumi.noob' methods implemented in the RnBeads package was applied $^{40,41}$. We removed probes containing an SNP in the assayed CpG dinucleotide, as well as those for which two or more SNPs were located in the probe sequence ${ }^{7}$. We removed probes not mapping uniquely to the human reference genome (hg19) allowing for one mismatch under the criteria of Price et al. ${ }^{42}$ Non-CpG targeting probes (Ch probes) and the probes included in the sex chromosomes were also removed ${ }^{43}$. Using the annotations provided by Illumina for the HumanMethylation450 platform, only probes located in the CpG islands and shores were kept for analysis in this study. The $\mathrm{R}$ Linear Models for Microarray Data (Limma) package ${ }^{44}$ was used to compare $\beta$-values and to identify differentially methylated probes between cancer and adjacent normal tissues. $P$-values were calculated from the moderated $t$-statistics and multiple testing correction of the $P$-values was performed using Benjamini and Hochberg's method (false discovery rate), to identify differentially methylated probes. Microarray data were uploaded to the National Center for Biotechnology Information's Gene 
a

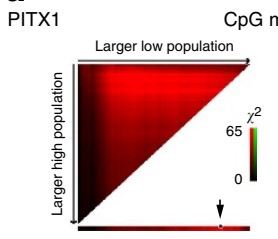

CpG methylation

b

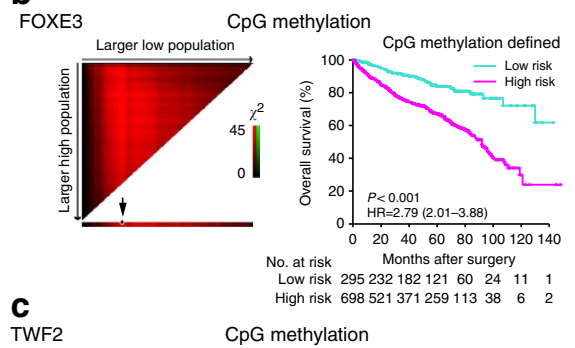

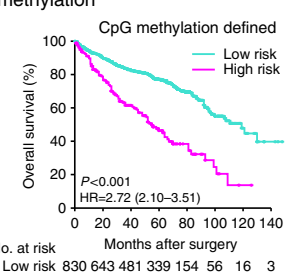
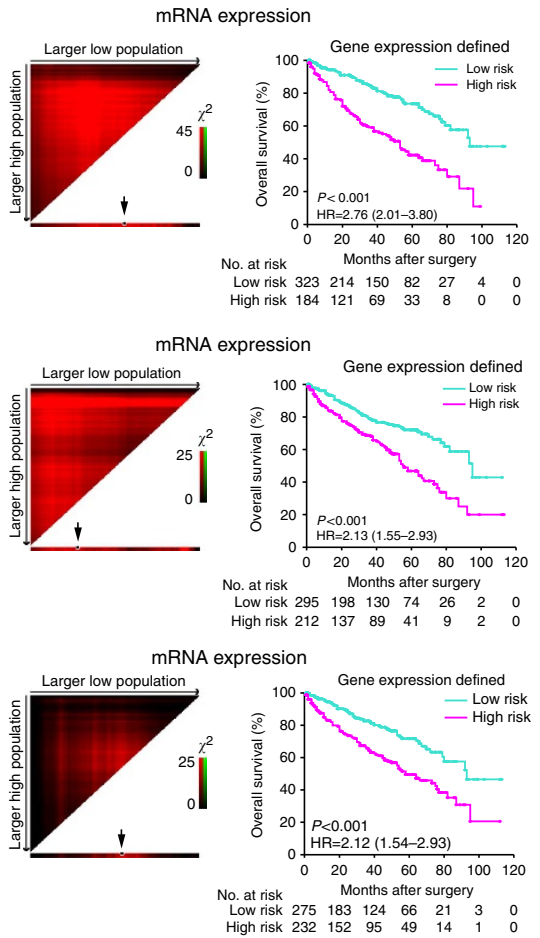

d

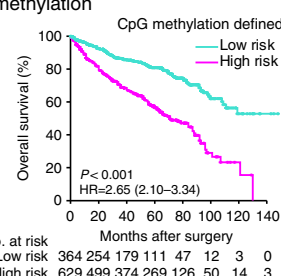

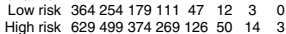

CpG methylation
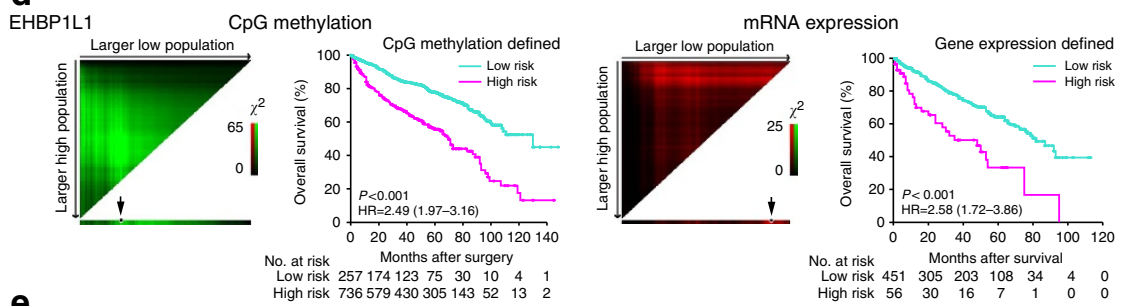

e

pG methylation
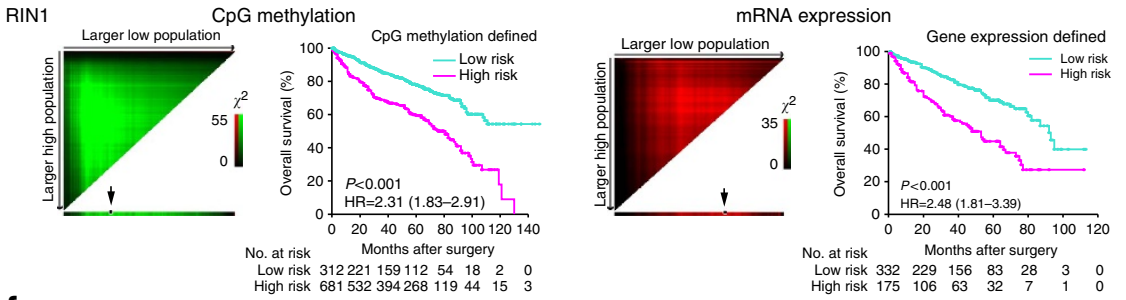

f
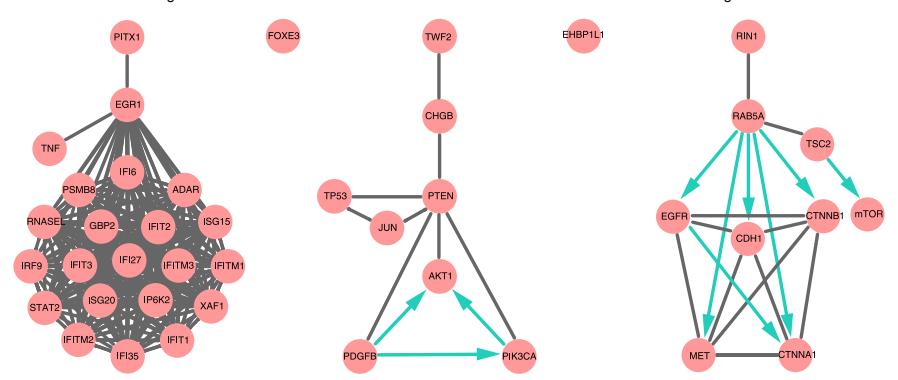

Figure 6 | X-tile plots of the genes that correspond to the five CpGs and network analyses. X-tile plots of the CpG methylation (993 patients in the entire cohort) and mRNA expression of the five genes (507 patients in the TCGA data set): (a) PITX1, (b) FOXE3, (c) TWF2, (d) EHBP1L1 and (e) RIN1. X-tile plots provide a single and intuitive method to assess the association between marker expression and survival, and automatically select the optimum cut point according to the highest $\chi^{2}$-value defined by Kaplan-Meier survival analysis and log-rank test. Colouration of the plot represents the strength of the association at each division, ranging from low (dark, black) to high (bright, red or green). Red represents inverse association between marker expression and survival, whereas green represents direct association between marker expression and survival. Each pixel represents an individual cutpoint where the number of patients in the group increases as progressed down for the high-expression group ('larger high population') or to the right for the low-expression group ('larger low population'). The dark dots (indicated by arrow) in the X-tile plots are the sites according to the highest $\chi^{2}$-value and are used as the cutoff points separating patients into high-risk and low-risk groups. (f) Network analyses of the genes that correspond to the five CpGs by cBioPortal. PITX1, TWF2 and RIN1 were predicted to have an impact on a diverse network of genes and pathways, as per the cBioPortal for Cancer Genomics network analysis tool. Black line means interactions between the two entities; blue arrow represents that the first entity controls a reaction that changes the state of the second entity. $\mathrm{HR}$, hazard ratio. 
Expression Omnibus (Series GSE61441, http://www.ncbi.nlm.nih.gov/geo/query/ acc.cgi?token=ufaxumuubrqxpgr\&acc=GSE61441).

Pyrosequencing. The methylation level of $\mathrm{CpG}$ sites was evaluated with pyrosequencing in the SYSU, MCHC and UTSW sets. DNA from paraffin-embedded tissue blocks was extracted from four sequential unstained sections, each $15 \mu \mathrm{m}$ thick. For each sample of tumour tissue, subsequent sections were stained with haematoxylin and eosin for histological confirmation of the presence $(>70 \%)$ of tumour cells. Genomic DNA was extracted with the QIAamp DNA FFPE Tissue Kit (Qiagen) following the manufacturer's recommendations. Bisulfite conversion was performed on $1 \mu \mathrm{g}$ of DNA with the EpiTect Bisulfite Kit (Qiagen). Twenty nanograms of converted DNA was used as a template in each subsequent PCR. Specific sets of primers for PCR amplification and sequencing were designed using the PyroMark Assay Design 2.0 software (Qiagen). All primer sequences are listed in Supplementary Table 2. PCRs were performed with the PyroMark PCR Kit (Qiagen) under the following conditions: $95^{\circ} \mathrm{C}$ for $15 \mathrm{~min}, 45$ cycles of $94^{\circ} \mathrm{C}$ for $30 \mathrm{~s}, 56^{\circ} \mathrm{C}$ for $30 \mathrm{~s}$ and $72^{\circ} \mathrm{C}$ for $30 \mathrm{~s}$, and an elongation step of $72^{\circ} \mathrm{C}$ for $10 \mathrm{~min}$. The success of amplification was assessed by $2 \%$ agarose gel electrophoresis. PCR products were pyrosequenced with the PyroMark Q24 pyrosequencer (Qiagen) according to the manufacturer's protocol (Pyro-Gold reagents). Output data were analysed using PyroMark Q24 2.0.6 Software (Qiagen), which calculates the CpG methylation value as the percentage $(\mathrm{mC} /[\mathrm{mC}+\mathrm{C}])$ for each $\mathrm{CpG}$ site, allowing quantitative comparisons. Controls to assess proper bisulfite conversion of the DNA were included in each run and sequencing controls were used to ensure the fidelity of the measurements.

TCGA data and network analysis. For the TCGA set, clinical data, CpG methylation value (level 3 data, Infinium HumanMethylation450) and mRNA expression (level 3 data, RNA-seq Version 2 Illumina) were downloaded from the TCGA data portal (http://tcga-data.nci.nih.gov/tcga/) on 1 October 2014. The clinical data included 512 retrospectively identified patients who underwent radical or partial nephrectomy between 1998 and 2010 for sporadic $\mathrm{ccRCC}^{45}$. Of the 512 patients, CpG methylation data were available for 298 patients and mRNA expression data were available for 507 patients. Of the 298 patients, VHL, PBRM1 and BAP1 gene mutation data were available for 242 (Supplementary Fig. 6). The cBioPortal for Cancer Genomics (http://cbioportal.org) network was used to search for pathways and interactions that might be linked to genes that correspond to the identified CpG sites in $\mathrm{ccRCC}^{46}$

Intratumour heterogeneity. ITH was investigated by extracting DNA samples from morphologically distinct regions within the tumours of 23 patients with ccRCC treated between 2011 and 2013 at the First Affiliated Hospital of SYSU (FFPE specimens; three different regions coded as R1, R2 and R3; Supplementary Fig.4). Methylation of the five $\mathrm{CpG}$ sites was detected with pyrosequencing. The s.d. and $\mathrm{CV}$ were used to describe the inter-sample variability of $\mathrm{CpG}$ methylation between the 23 ccRCCs and the intra-sample variability between different regions.

Statistical analysis. The goal of this study was to identify prognostic classifier that predicts overall survival. This is defined as the time between surgery and death or the last follow-up date. Volcano plot analysis was used to select $\mathrm{CpG}$ sites based on absolute fold change in combination with $t$-test $P$-values. LASSO logistic regression analysis was used to identify the candidate $\mathrm{CpG}$ sites with non-zero coefficients in the discovery set. LASSO Cox regression analysis was used to select the prognostic markers of the candidate $\mathrm{CpG}$ sites and to construct a multi-CpG-based classifier for predicting the overall survival of patients with ccRCC in the SYSU set. We used the Kaplan-Meier method to analyse the correlation between variables and overall survival, and we used the log-rank test to compare survival curves. Multivariate survival analysis was performed using the Cox regression model. X-tile plots were used to generate the optimum cutoff point for continuous variables according to the highest $\chi^{2}$-value defined by Kaplan-Meier survival analysis and log-rank test ${ }^{47}$. $\mathrm{X}$-tile plots were created with X-tile software version 3.6.1 (Yale University School of Medicine, New Haven, CT, USA) and all the other statistical tests were performed with R software version 3.0.3 (R Foundation for Statistical Computing, Vienna, Austria). Statistical significance was set at 0.05 .

LASSO regression analysis. The high dimensionality of microarray-based experiments in contrast to the small number of samples easily leads to overfitting. Regularized linear models such as logistic regression with LASSO penalty are popular solutions to fitting sparse models in which only a small subset of features plays a role ${ }^{48}$. LASSO can be used with high-dimensional data for optimal selection of genes with a strong diagnostic or prognostic value and low correlation among each other to prevent overfitting ${ }^{49-52}$. LASSO is a form of regularized or 'penalized' regression where L1 regularization is introduced into the standard multiple linear regression procedure using a compound cost function to optimize the regression coefficients. LASSO regression shrinks the coefficient estimates towards zero, with the degree of shrinkage depending on an additional parameter, $\lambda$. In this way, coefficient estimates can be forced to be exactly zero, thereby effectively eliminating a number of variables. We adopted the LASSO regression model to achieve shrinkage and variable selection simultaneously. Ten-time cross-validations were used to determine the optimal values of $\lambda$ (refs 51-53). We choose $\lambda$ via $1-$ s.e. criteria, that is, the optimal $\lambda$ is the largest value for which the partial likelihood deviance is within 1 s.e. of the smallest value of partial likelihood deviance ${ }^{24}$. We used R software version 3.0.3 (R Foundation for Statistical Computing) and the 'glmnet' package to perform LASSO regression analysis.

\section{References}

1. Ljungberg, B. et al. EAU guidelines on renal cell carcinoma: 2014 update. Eur. Urol. 67, 913-924 (2015).

2. Zigeuner, R. et al. External validation of the Mayo Clinic stage, size, grade, and necrosis (SSIGN) score for clear-cell renal cell carcinoma in a single European centre applying routine pathology. Eur. Urol. 57, 102-109 (2010).

3. Ficarra, V. et al. The 'Stage, Size, Grade and Necrosis' score is more accurate than the University of California Los Angeles Integrated Staging System for predicting cancer-specific survival in patients with clear cell renal cell carcinoma. BJU Int. 103, 165-170 (2009)

4. Brock, M. V. et al. DNA methylation markers and early recurrence in stage I lung cancer. N. Engl. J. Med. 358, 1118-1128 (2008).

5. Castelo-Branco, P. et al. Methylation of the TERT promoter and risk stratification of childhood brain tumours: an integrative genomic and molecular study. Lancet Oncol. 14, 534-542 (2013).

6. Esteller, M. Relevance of DNA methylation in the management of cancer. Lancet Oncol. 4, 351-358 (2003).

7. Sandoval, J. et al. A prognostic DNA methylation signature for stage I non-small-cell lung cancer. J. Clin. Oncol. 31, 4140-4147 (2013).

8. Cancer Genome Atlas Research Network. Genomic and epigenomic landscapes of adult de novo acute myeloid leukemia. N. Engl. J. Med. 368, 2059-2074 (2013).

9. Ricketts, C. J. et al. Genome-wide $\mathrm{CpG}$ island methylation analysis implicates novel genes in the pathogenesis of renal cell carcinoma. Epigenetics 7, 278-290 (2012).

10. Lasseigne, B. N. et al. DNA methylation profiling reveals novel diagnostic biomarkers in renal cell carcinoma. BMC Med. 12, 235 (2014).

11. Arai, E. et al. Multilayer-omics analysis of renal cell carcinoma, including the whole exome, methylome and transcriptome. Int. J. Cancer 135, 1330-1342 (2014).

12. Ibragimova, I. et al. Genome-wide promoter methylome of small renal masses. PLoS ONE 8, e77309 (2013).

13. Kratz, J. R. et al. A practical molecular assay to predict survival in resected non-squamous, non-small-cell lung cancer: development and international validation studies. Lancet 379, 823-832 (2012).

14. van de Vijver, M. J. et al. A gene-expression signature as a predictor of survival in breast cancer. N. Engl. J. Med. 347, 1999-2009 (2002).

15. Liu, N. et al. Prognostic value of a microRNA signature in nasopharyngeal carcinoma: a microRNA expression analysis. Lancet Oncol. 13, 633-641 (2012).

16. Yoon, K. A. et al. Genetic variations associated with postoperative recurrence in stage I non-small cell lung cancer. Clin. Cancer Res. 20, 3272-3279 (2014).

17. Buyse, M. et al. Validation and clinical utility of a 70-gene prognostic signature for women with node-negative breast cancer. J. Natl Cancer Inst. 98, 1183-1192 (2006).

18. De Sousa, E. M. F. et al. Poor-prognosis colon cancer is defined by a molecularly distinct subtype and develops from serrated precursor lesions. Nat. Med. 19, 614-618 (2013).

19. Arai, E. et al. Single-CpG-resolution methylome analysis identifies clinicopathologically aggressive $\mathrm{CpG}$ island methylator phenotype clear cell renal cell carcinomas. Carcinogenesis 33, 1487-1493 (2012).

20. Simon, R. \& Altman, D. G. Statistical aspects of prognostic factor studies in oncology. Br. J. Cancer 69, 979-985 (1994).

21. Joseph, F., Hair, J., Anderson, R. E., Tatham, R. L. \& Black, W. C. Multivariate Data Analysis, 4th edn (Prentice-Hall, Inc., 1995).

22. Tibshirani, R. The lasso method for variable selection in the Cox model. Stat. Med. 16, 385-395 (1997)

23. Zhang, H. H. \& Lu, W. Adaptive Lasso for Cox's proportional hazards model Biometrika 94, 691-703 (2007).

24. Zhang, J. X. et al. Prognostic and predictive value of a microRNA signature in stage II colon cancer: a microRNA expression analysis. Lancet Oncol. 14, 1295-1306 (2013).

25. Gerlinger, M. et al. Intratumor heterogeneity and branched evolution revealed by multiregion sequencing. N. Engl. J. Med. 366, 883-892 (2012).

26. Gulati, S. et al. Systematic evaluation of the prognostic impact and intratumour heterogeneity of clear cell renal cell carcinoma biomarkers. Eur. Urol. 66, 936-948 (2014).

27. Barry, W. T. et al. Intratumor heterogeneity and precision of microarray-based predictors of breast cancer biology and clinical outcome. J. Clin. Oncol. 28, 2198-2206 (2010).

28. Zhao, H. et al. Gene expression profiling predicts survival in conventional renal cell carcinoma. PLoS Med. 3, e13 (2006). 
29. Kosari, F. et al. Clear cell renal cell carcinoma: gene expression analyses identify a potential signature for tumor aggressiveness. Clin. Cancer Res. 11, 5128-5139 (2005).

30. Brooks, S. A. et al. ClearCode34: A prognostic risk predictor for localized clear cell renal cell carcinoma. Eur. Urol. 66, 77-84 (2014).

31. Escudier, B. J. et al. Validation of a 16-gene signature for prediction of recurrence after nephrectomy in stage I-III clear cell renal cell carcinoma (ccRCC). ASCO Meeting Abstracts 32, 4502 (2014).

32. Chatterton, Z. et al. Validation of DNA methylation biomarkers for diagnosis of acute lymphoblastic leukemia. Clin. Chem. 60, 995-1003 (2014).

33. Bell, A., Bell, D., Weber, R. S. \& El-Naggar, A. K. CpG island methylation profiling in human salivary gland adenoid cystic carcinoma. Cancer 117, 2898-2909 (2011).

34. Milstein, M. et al. RIN1 is a breast tumor suppressor gene. Cancer Res. 67, 11510-11516 (2007)

35. Yamada, S. et al. Identification of twinfilin-2 as a factor involved in neurite outgrowth by RNAi-based screen. Biochem. Biophys. Res. Commun. 363, 926-930 (2007).

36. West, J., Widschwendter, M. \& Teschendorff, A. E. Distinctive topology of age-associated epigenetic drift in the human interactome. Proc. Natl Acad. Sci. USA 110, 14138-14143 (2013).

37. Cheng, C. P. et al. Network-based analysis identifies epigenetic biomarkers of esophageal squamous cell carcinoma progression. Bioinformatics 30, 3054-3061 (2014).

38. Dick, K. J. et al. DNA methylation and body-mass index: a genome-wide analysis. Lancet 383, 1990-1998 (2014).

39. Assenov, Y. et al. Comprehensive analysis of DNA methylation data with RnBeads. Nat. Methods 11, 1138-1140 (2014).

40. Teschendorff, A. E. et al. A beta-mixture quantile normalization method for correcting probe design bias in Illumina Infinium $450 \mathrm{k}$ DNA methylation data. Bioinformatics 29, 189-196 (2013).

41. Triche, Jr T. J., Weisenberger, D. J., Van Den Berg, D., Laird, P. W. \& Siegmund, K. D. Low-level processing of Illumina Infinium DNA Methylation BeadArrays. Nucleic Acids Res. 41, e90 (2013).

42. Price, M. E. et al. Additional annotation enhances potential for biologicallyrelevant analysis of the Illumina Infinium HumanMethylation450 BeadChip array. Epigenetics Chromatin 6, 4 (2013).

43. Chen, Y. A. et al. Discovery of cross-reactive probes and polymorphic CpGs in the Illumina Infinium HumanMethylation450 microarray. Epigenetics 8, 203-209 (2013).

44. Gentleman, R., Carey, V., Huber, W., Irizarry, R. \& Dudoit, S. Bioinformatics and Computational Biology Solutions Using $R$ and Bioconductor (Statistics for Biology and Health) (Springer-Verlag, Inc., 2005).

45. Cancer Genome Atlas Research Network. Comprehensive molecular characterization of clear cell renal cell carcinoma. Nature 499, 43-49 (2013).

46. Gao, J. et al. Integrative analysis of complex cancer genomics and clinical profiles using the cBioPortal. Sci. Signal. 6, pl1 (2013).

47. Camp, R. L., Dolled-Filhart, M. \& Rimm, D. L. X-tile: a new bio-informatics tool for biomarker assessment and outcome-based cut-point optimization. Clin. Cancer Res. 10, 7252-7259 (2004).
48. Tibshirani, R. Regression shrinkage and selection via the Lasso. J. R. Stat. Soc. Ser. B 58, 267-288 (1996).

49. Goeman, J. J. L1 penalized estimation in the Cox proportional hazards model. Biom. J. 52, 70-84 (2010).

50. Gui, J. \& Li, H. Penalized Cox regression analysis in the high-dimensional and low-sample size settings, with applications to microarray gene expression data. Bioinformatics 21, 3001-3008 (2005).

51. Sveen, A. et al. ColoGuidePro: a prognostic 7-gene expression signature for stage III colorectal cancer patients. Clin. Cancer Res. 18, 6001-6010 (2012).

52. Olk-Batz, C. et al. Aberrant DNA methylation characterizes juvenile myelomonocytic leukemia with poor outcome. Blood 117, 4871-4880 (2011).

53. Kohavi, R. In Proceedings of the 14th International Joint Conference on Artificial Intelligence, Vol 2 (Morgan Kaufmann Publishers Inc., 1995).

\section{Acknowledgements}

The study was supported by grants from the National Natural Science Foundation of China (81572905, 81372730, 81225018 and 81372357) and the Guangdong Provincial Science and Technology Foundation (2014B020212015). We thank the TCGA for their efforts and providing data.

\section{Author contributions}

J.H.L. designed the study. A.H., K.J.W., H.W.Z., Z.L.Z., L.Y.Z., Z.H.C., Y.H.Y., Z.R.W., F.J.Z., L.S., Q.Z. Liu, Z.L.G., D.L.H., W.C., J.T.H. and V.M. obtained and assembled data J.H.W., A.H., K.J.W., H.W.Z., P.K., Z.L.Z., L.Y.Z., Z.H.C., Y.Y.Z., J.C.Z., B.W., M.Y.C., D.X., B.L., C.X.L., P.X.L., Q.Z. Li and J.H.L. analysed and interpreted the data. J.H.W., A.H. and J.H.L. wrote the report, which was edited by all authors, who have approved the final version. J.H.L., W.C. and D.X. are the guarantors.

\section{Additional information}

Accession codes: Methylation array data have been deposited in Gene Expression Omnibus database under accession code GSE61441.

Supplementary Information accompanies this paper at http://www.nature.com/ naturecommunications

Competing financial interests: The authors declare no competing financial interests.

Reprints and permission information is available online at http://npg.nature.com/ reprintsandpermissions/

How to cite this article: Wei, J.-H. et al. A CpG-methylation-based assay to predict survival in clear cell renal cell carcinoma. Nat. Commun. 6:8699 doi: 10.1038/ncomms9699 (2015).

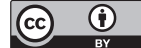

This work is licensed under a Creative Commons Attribution 4.0 International License. The images or other third party material in this article are included in the article's Creative Commons license, unless indicated otherwise in the credit line; if the material is not included under the Creative Commons license, users will need to obtain permission from the license holder to reproduce the material To view a copy of this license, visit http://creativecommons.org/licenses/by/4.0/ 